\title{
Sunki Mandarin vs Poncirus trifoliata Hybrids as Rootstocks for Pera Sweet Orange
}

\author{
Evandro Henrique Schinor ${ }^{1}$, Mariângela Cristofani-Yaly ${ }^{1}$, Marinês Bastianel ${ }^{1}$ \& Marcos Antonio Machado $^{1}$ \\ ${ }^{1}$ Centro APTA Citros "Sylvio Moreira”, Instituto Agronômico, Cordeirópolis, SP, Rodovia Anhanguera, km 158, \\ CP 4, CEP 13490-970, Brasil \\ Correspondence: Evandro Henrique Schinor, Centro APTA Citros "Sylvio Moreira", Instituto Agronômico, \\ Cordeirópolis, SP, Rodovia Anhanguera, $\mathrm{km} \mathrm{158,} \mathrm{CP} \mathrm{4,} \mathrm{CEP} \mathrm{13490-970,} \mathrm{Brasil.} \mathrm{E-mail:}$ \\ evandro@centrodecitricultura.br
}

Received: March 2, 2013 Accepted: April 5, 2013 Online Published: May 15, 2013

doi:10.5539/jas.v5n6p190 URL: http://dx.doi.org/10.5539/jas.v5n6p190

\begin{abstract}
Obtaining new rootstocks that have resistance to biotic and abiotic factors is one of the main goals of breeding programs for citrus. This study evaluated the performance of 42 hybrids of Sunki mandarin (Citrus sunki Hort. ex Tanaka) vs. Poncirus trifoliata cv. Rubidoux, as rootstock for Pera sweet orange. The experiment was conducted in Colômbia, São Paulo, Brazil, in randomized blocks with three replications in a spacing of $6.0 \mathrm{~m} \times 3.5 \mathrm{~m}$. The trees were seven years old and the experiment was conducted without irrigation. We quantified the variables height, diameter and canopy volume, and production of plants, besides the physico-chemical analysis of fruits. After the natural period of drought in the region, the trees were evaluated for resistance to drought, with scale ranging from 1 to 3. Plants were also evaluated for symptoms of citrus sudden death and compatibility canopy/rootstock. Differences were observed in height, diameter and canopy volume of Pera sweet orange grafted on 42 hybrids of Sunki mandarin x Poncirus trifoliata (TSxPT). Differences were observed in the physico-chemical characteristics of fruits of Pera sweet orange grafted on different hybrids TSxPT. Different degrees of drought tolerance were observed and six hybrids were resistant. The hybrids TSxPT 245 and 254 showed incompatibility with variety of Pera sweet orange.
\end{abstract}

Keywords: citrandarin, Citrus sinensis, genetic breeding

\section{Introduction}

Hybrids from crosses of Sunki (Citrus sunki hort. ex Tanaka) or Cleopatra (C. reshni hort. ex Tanaka) mandarins with Poncirus trifoliata (L.) Raf. are called citrandarins, in which we want to gather the advantages presented by the mandarins, as lower susceptibility to citrus decline, to exocortis viroid and calcareous soils, with those of $P$. trifoliata, as resistance to citrus tristeza virus, to root rot caused by Phytophthora, frost, and induce the formation of smaller plants (Blumer \& Pompeu Junior, 2005; Pompeu Junior \& Blumer, 2009, 2011).

Today, with the emergence and spread of Huanglongbing (HLB) in the State of São Paulo, Brazil, smaller citrus plants are desirable because they have some advantages over larger ones. Smaller plants may increase the efficiency of inspection and hence the control of pests and diseases, reduce production costs, increase security at harvest, produce more fruit per cubic meter of canopy and allow high planting densities, enabling greater yield per area (Pompeu Junior, 2001; Pompeu Junior \& Blumer, 2009).

In the State of São Paulo, most citrus orchards are not irrigated and flowering usually occurs between the months of August and September, a period of lower rainfall, making it necessary to use rootstocks tolerant to drought, an important characteristics of Rangpur lime ( $C$. limonia Osbeck), conferring earliness and high yield to canopy varieties grafted on it (Pompeu Junior, 2005), however, the Rangpur lime is susceptible to citrus decline (Rodriguez et al., 1979) and citrus sudden death (Fernandes \& Gimenes-Bassanezi, 2001).

Diversification in the use of rootstocks in citrus can overcome abiotic and biotic problems and result in gains in productivity. The use of limited number of canopy / rootstock generated great adversity of brazilian citriculture, such as root rot caused by Phytophthora (Alencar, 1941), the citrus tristeza virus (Bittancourt, 1940), the decline of citrus and citrus sudden death. 
With so many adversities in citrus, obtaining new rootstocks for diversification in orchards, is one of the main objectives of the breeding programs of citrus. The Centro APTA Citros 'Sylvio Moreira'/Instituto Agronômico is developing a breeding program of rootstocks having as goal to maintain and extend the studies of selection of new rootstocks, either by obtaining hybrids or the use of existing accesses of the Active Germplasm Bank of citrus.

This study aimed to evaluate the performance of 42 hybrids of Sunki mandarin vs Poncirus trifoliata cv. Rubidoux as rootstock for Pera sweet orange [C. sinensis (L.) Osbeck].

\section{Materials and Methods}

The work was conducted at Muriti farm property of the Fischer group, in the municipality of Colômbia, São Paulo State, Brazil, geographic coordinates $24^{\circ} 17^{\prime} \mathrm{S}$ and $48^{\circ} 24^{\prime} \mathrm{W}$, altitude of 480 meters and climate type aw, according to the Köppen classification (Ortolani, Júnior, \& Alfonsi, 1991). The average annual rainfall is 1429.1 $\mathrm{mm}$ and the average annual temperature is $23.8^{\circ} \mathrm{C}$, with an average maximum temperature equal to $30.7^{\circ} \mathrm{C}$ and minimum of $17.0^{\circ} \mathrm{C}$ (CEPAGRI, 2011).

The experiment was arranged in randomized complete block design. It was implemented in 2003, in a spacing of $6.0 \mathrm{~m}$ x $3.5 \mathrm{~m}$, initially consisting of 111 hybrids of Sunki mandarin vs $P$. trifoliata cv. Rubidoux (TSxPT) grafted with Pera sweet orange with three replications and conducted without irrigation. Forty-two of these TSxPT hybrids were selected for purposes of evaluations in this work.

The height and diameter of the tree canopy were determined from measurements using a ruler graduated in centimeters. We calculated the volume of the canopy using the function (Mendel, 1956):

$$
V=2 / 3 \pi R^{2} H
$$

Where,

$$
\begin{aligned}
& \mathrm{V}=\text { canopy volume }\left(\mathrm{m}^{3}\right) ; \\
& \mathrm{R}=\text { the canopy radius }(\mathrm{m}) ; \\
& \mathrm{H}=\text { plant height }(\mathrm{m}) .
\end{aligned}
$$

We evaluated also the fruit yield $\left(\mathrm{kg}\right.$ tree $\left.^{-1}\right)$ and calculated the values of productivity $\left(\mathrm{kg} \mathrm{m}^{-3}\right)$, in the years 2007 and 2010.

For physical and chemical analysis, samples of five fruits were collected of each plant in the second week of October 2009 and sent to the Quality and Postharvest Laboratory of Centro APTA Citros 'Sylvio Moreira'/Instituto Agronômico, Cordeirópolis, SP. Measurements of height $(\mathrm{H})$ and diameter (D) of the fruits were made by direct reading of each sample, with the help of ruler graduated in centimeters, and from these values, we calculated the relation $\mathrm{H} / \mathrm{D}$ of the fruit. The total mass of the fruits was measured in grams, and the characteristics of juice were obtained as follows: the juice yield was determined after crushing of five fruits of each sample in extractor OIC model OTTO 1800 andcalculated using the relation between juice/fruit mass and expressed as a percentage, the soluble solids $\left({ }^{\circ}\right.$ Brix)content was obtained by direct reading using refractometer B \& S model RFM 330, , the juice acidity was determined by titrating an aliquot of $25 \mathrm{~mL}$ of juice to $\mathrm{pH} 8.2$, using a solution of sodium hydroxide $(\mathrm{NaOH})$ with normality of 0.3125 $\mathrm{N}$, and phenolphthalein as indicator. The ratio was calculated using the relation between soluble solids: acidity and the technological indexwas obtained according Di Giorgi et al. (1990) and expressed in Kg of total soluble solids (TSS) per box with $40.8 \mathrm{~kg}$ of fruit

In the years 2007 and 2010, after about 90 days of drought in the region of the experiment, the plants were evaluated for tolerance to drought, assigning scores ranging from 1 to 3 , and score 1 to plant showing highly susceptibity, score 2 for moderately susceptible plant and score 3 to tolerant plants.

We also evaluated the compatibility between the scion variety (Pera) and each rootstock studied (TSxPT hybrids), identifying incompatible in combinations that had a line of gum or necrosis observed after removal of the bark of the trunk, in the region of grafting (Nauriyal, Shannon, \& Frolich, 1958). It is known that Pera sweet orange is incompatible with trifoliate orange and some of its hybrids (Pompeu Junior, 2005).

The resistance of citrus sudden death was evaluated by withdrawing part of the cortex, evaluating the presence of symptoms characteristic of the disease, ie, yellow-orange color in the internal tissues of the rootstock below the graft region, contrasting with the color of the inner tissue of the bark of the canopy. These tissues yellowish correspond for the most part to the phloem vessels that are obstructed and degenerate (Gimenes-Fernandes \& Bassanezi, 2001). 
For statistical analysis of the studied variables, we used the parametric test Knott Scott (Scott \& Knott, 1974), which separates the means by comparisons between groups of data, calculated by means of the statistical program SISVAR (Ferreira, 2008).

\section{Results and Discussion}

The results showed that the hybrids TSxPT 14, 26, 110 and 137 were those that produce less height $(<2.0 \mathrm{~m})$, smaller diameter $(<2.2 \mathrm{~m})$ and, consequently, lower canopy $\left(\leq 5.0 \mathrm{~m}^{3}\right)$ for plants of Pera sweet orange evaluated at seven years after planting. These rootstocks can be used for high planting density. Moreover, some hybrids evaluated in this study (TSxPT 92, 132, 142 and 155) induced high vigor to Pera sweet orange plants, with trees exceeding $3.0 \mathrm{~m}$ in height, diameter above $3.2 \mathrm{~m}$ and canopy volume greater than $17.0 \mathrm{~m}^{3}$ (Table 1 and Figure 1 ).

According Pompeu Junior (2005), rootstocks inducing dwarfism are usually selections or hybrids of $P$. trifoliata, like the Flying Dragon, which can be considered a genetically dwarfing rootstock, inducing the formation of mature plants with height less than $2.5 \mathrm{~m}$, even under adverse weather and soil conditions, with or without irrigation. Pompeu Junior \& Blumer (2009) reported the induction of dwarfism in Valencia sweet orange plants grafted on citrandarin (Cleopatra mandarin x P. trifoliata cv. Christian - 712), where they found plants with an average height of $1.90 \mathrm{~m}$ at 16 years.

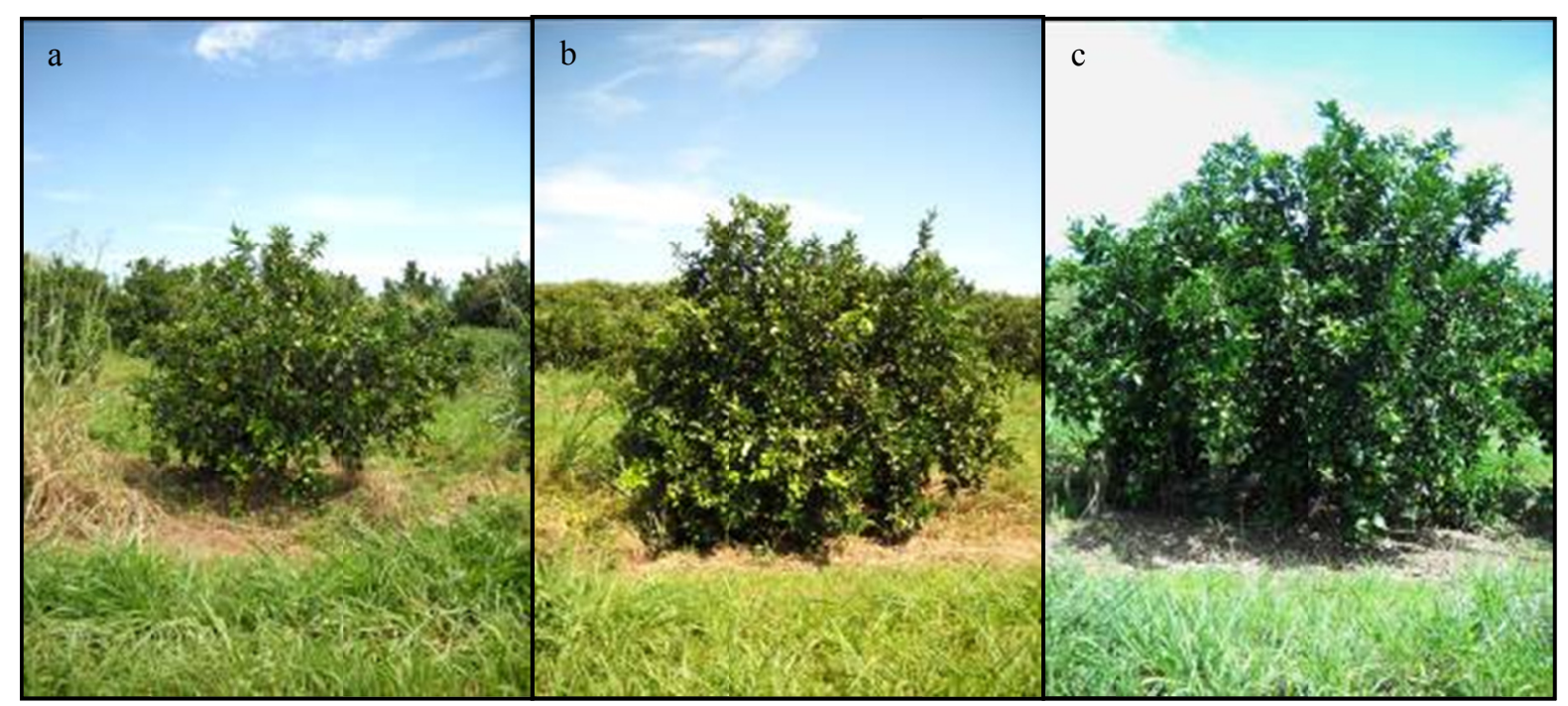

Figure 1. Pera sweet orange grafted on hybrids of Sunki mandarin x Poncirus trifoliata (TSxPT) presenting different scion sizesa) Hybrid TSxPT 137 - dwarf (1.97 m high), b) Hybrid TSxPT 106 - half-dwarf (2.50 m high), c) Hybrid TSxPT 108 - tall (2.90 m high) (Colômbia, SP, Brazil, 2010). 
Table 1. Height, diameter and canopy volume of plants of Pera sweet orange grafted on hybrids of Sunki mandarin x Poncirus trifoliata. Colômbia, SP, Brazil, 2007-2010

\begin{tabular}{|c|c|c|c|c|c|c|}
\hline \multirow{2}{*}{ Hybrids } & \multicolumn{2}{|c|}{ Height (m) } & \multicolumn{2}{|c|}{ Diameter (m) } & \multicolumn{2}{|c|}{ Volume $\left(\mathrm{m}^{3}\right)$} \\
\hline & 2007 & 2010 & 2007 & 2010 & 2007 & 2010 \\
\hline TSxPT 7 & $1.75 \mathrm{c}$ & $2.33 \mathrm{~b}$ & $1.66 \mathrm{~b}$ & $2.70 \mathrm{c}$ & $2.52 \mathrm{a}$ & $9.14 \mathrm{~b}$ \\
\hline TSxPT 14 & $1.10 \mathrm{a}$ & $1.40 \mathrm{a}$ & $1.23 \mathrm{a}$ & $1.93 \mathrm{a}$ & $0.91 \mathrm{a}$ & $2.79 \mathrm{a}$ \\
\hline TSxPT 16 & $1.73 \mathrm{c}$ & $2.50 \mathrm{c}$ & $1.55 \mathrm{a}$ & $2.35 \mathrm{~b}$ & $2.29 \mathrm{a}$ & $7.27 \mathrm{~b}$ \\
\hline TSxPT 17 & $1.75 \mathrm{c}$ & $2.60 \mathrm{c}$ & $1.77 \mathrm{~b}$ & $2.83 \mathrm{c}$ & $3.01 \mathrm{~b}$ & $10.95 \mathrm{c}$ \\
\hline TSxPT 23 & $1.71 \mathrm{c}$ & $2.77 \mathrm{c}$ & $1.46 \mathrm{a}$ & $2.83 \mathrm{c}$ & $1.93 \mathrm{a}$ & $11.69 \mathrm{c}$ \\
\hline TSxPT 26 & $1.34 \mathrm{~b}$ & $1.80 \mathrm{a}$ & $1.46 \mathrm{a}$ & $1.93 \mathrm{a}$ & $1.53 \mathrm{a}$ & $3.57 \mathrm{a}$ \\
\hline TSxPT 38 & $1.70 \mathrm{c}$ & $2.50 \mathrm{c}$ & $1.67 \mathrm{~b}$ & $2.70 \mathrm{c}$ & $2.54 \mathrm{a}$ & $9.56 \mathrm{~b}$ \\
\hline TSxPT 42 & $1.92 \mathrm{c}$ & $2.75 \mathrm{c}$ & $1.57 \mathrm{a}$ & $2.75 \mathrm{c}$ & $2.51 \mathrm{a}$ & $11.80 \mathrm{c}$ \\
\hline TSxPT 54 & $2.34 \mathrm{~d}$ & $2.95 \mathrm{~d}$ & $2.05 \mathrm{~b}$ & $3.10 \mathrm{c}$ & $5.14 \mathrm{~b}$ & $14.94 \mathrm{~d}$ \\
\hline TSxPT 56 & $1.83 \mathrm{c}$ & $2.70 \mathrm{c}$ & $1.57 \mathrm{a}$ & $2.47 \mathrm{~b}$ & $2.39 \mathrm{a}$ & $8.57 \mathrm{~b}$ \\
\hline TSxPT 68 & $1.28 \mathrm{~b}$ & $2.17 \mathrm{~b}$ & $1.29 \mathrm{a}$ & $2.33 \mathrm{~b}$ & $1.20 \mathrm{a}$ & $6.20 \mathrm{~b}$ \\
\hline TSxPT 70 & $1.78 \mathrm{c}$ & $2.33 \mathrm{~b}$ & $1.53 \mathrm{a}$ & $2.20 \mathrm{~b}$ & $2.40 \mathrm{a}$ & $6.54 \mathrm{~b}$ \\
\hline TSxPT 86 & $2.22 \mathrm{~d}$ & $2.75 \mathrm{c}$ & $2.06 \mathrm{~b}$ & $2.95 \mathrm{c}$ & $4.92 \mathrm{~b}$ & $12.54 \mathrm{c}$ \\
\hline TSxPT 92 & $2.02 \mathrm{~d}$ & $3.23 \mathrm{~d}$ & $1.92 \mathrm{~b}$ & $3.30 \mathrm{c}$ & $4.04 \mathrm{~b}$ & $18.46 \mathrm{~d}$ \\
\hline TSxPT 101 & $1.82 \mathrm{c}$ & $2.80 \mathrm{c}$ & $1.76 \mathrm{~b}$ & $3.00 \mathrm{c}$ & $2.99 \mathrm{~b}$ & $13.19 \mathrm{c}$ \\
\hline TSxPT 106 & $1.91 \mathrm{c}$ & $2.50 \mathrm{c}$ & $1.85 \mathrm{~b}$ & $2.63 \mathrm{c}$ & $3.71 \mathrm{~b}$ & $9.53 \mathrm{~b}$ \\
\hline TSxPT 107 & $1.81 \mathrm{c}$ & $2.65 \mathrm{c}$ & $1.62 \mathrm{a}$ & $2.70 \mathrm{c}$ & $2.49 \mathrm{a}$ & $10.12 \mathrm{~b}$ \\
\hline TSxPT 108 & $1.98 \mathrm{~d}$ & $2.90 \mathrm{~d}$ & $1.82 \mathrm{~b}$ & $3.20 \mathrm{c}$ & $3.60 \mathrm{~b}$ & $15.59 \mathrm{~d}$ \\
\hline TSxPT 110 & $1.09 \mathrm{a}$ & $1.67 \mathrm{a}$ & $1.17 \mathrm{a}$ & $1.83 \mathrm{a}$ & $0.86 \mathrm{a}$ & $2.95 \mathrm{a}$ \\
\hline TSxPT 119 & $2.02 \mathrm{~d}$ & $2.95 \mathrm{~d}$ & $1.56 \mathrm{a}$ & $3.00 \mathrm{c}$ & $2.58 \mathrm{a}$ & $13.87 \mathrm{c}$ \\
\hline TSxPT 121 & $1.56 \mathrm{~b}$ & $2.25 \mathrm{~b}$ & $1.52 \mathrm{a}$ & $2.60 \mathrm{c}$ & $2.09 \mathrm{a}$ & $8.32 \mathrm{~b}$ \\
\hline TSxPT 124 & $2.11 \mathrm{~d}$ & $2.93 \mathrm{~d}$ & $1.94 \mathrm{~b}$ & $2.67 \mathrm{c}$ & $4.30 \mathrm{~b}$ & $11.11 \mathrm{c}$ \\
\hline TSxPT 126 & $1.92 \mathrm{c}$ & $3.00 \mathrm{~d}$ & $1.77 \mathrm{~b}$ & $3.10 \mathrm{c}$ & $3.15 \mathrm{~b}$ & $15.10 \mathrm{~d}$ \\
\hline TSxPT 128 & $2.10 \mathrm{~d}$ & $2.90 \mathrm{~d}$ & $1.53 \mathrm{a}$ & $2.55 \mathrm{~b}$ & $2.63 \mathrm{a}$ & $9.91 \mathrm{~b}$ \\
\hline TSxPT 132 & $2.11 \mathrm{~d}$ & $3.03 \mathrm{~d}$ & $1.85 \mathrm{~b}$ & $3.33 \mathrm{c}$ & $3.89 \mathrm{~b}$ & $17.79 \mathrm{~d}$ \\
\hline TSxPT 136 & $1.61 \mathrm{c}$ & $2.30 \mathrm{~b}$ & $1.37 \mathrm{a}$ & $2.27 \mathrm{~b}$ & $1.64 \mathrm{a}$ & $6.27 \mathrm{~b}$ \\
\hline TSxPT 137 & $1.16 \mathrm{a}$ & $1.97 \mathrm{~b}$ & $1.39 \mathrm{a}$ & $2.20 \mathrm{~b}$ & $1.16 \mathrm{a}$ & $5.01 \mathrm{a}$ \\
\hline TSxPT 139 & $1.76 \mathrm{c}$ & $2.25 \mathrm{~b}$ & $1.70 \mathrm{~b}$ & $2.85 \mathrm{c}$ & $3.29 \mathrm{a}$ & $10.08 \mathrm{~b}$ \\
\hline TSxPT 142 & $1.85 \mathrm{c}$ & $3.07 \mathrm{~d}$ & $1.80 \mathrm{~b}$ & $3.27 \mathrm{c}$ & $3.19 \mathrm{~b}$ & $17.15 \mathrm{~d}$ \\
\hline TSxPT 143 & $1.90 \mathrm{c}$ & $2.45 \mathrm{c}$ & $1.79 \mathrm{~b}$ & $2.50 \mathrm{~b}$ & $3.21 \mathrm{~b}$ & $8.02 \mathrm{~b}$ \\
\hline TSxPT 148 & $1.65 \mathrm{c}$ & $2.37 \mathrm{~b}$ & $1.72 \mathrm{~b}$ & $2.70 \mathrm{c}$ & $2.59 \mathrm{a}$ & $9.02 \mathrm{~b}$ \\
\hline TSxPT 152 & $1.97 \mathrm{~d}$ & $2.67 \mathrm{c}$ & $1.62 \mathrm{a}$ & $3.03 \mathrm{c}$ & $2.72 \mathrm{a}$ & $12.89 \mathrm{c}$ \\
\hline TSxPT 155 & $2.03 \mathrm{~d}$ & $3.13 \mathrm{~d}$ & $2.07 \mathrm{~b}$ & $3.40 \mathrm{c}$ & $4.63 \mathrm{~b}$ & $19.28 \mathrm{~d}$ \\
\hline TSxPT 166 & $2.09 \mathrm{~d}$ & $2.73 \mathrm{c}$ & $1.85 \mathrm{~b}$ & $2.80 \mathrm{c}$ & $3.75 \mathrm{~b}$ & $11.76 \mathrm{c}$ \\
\hline TSxPT 168 & $1.95 \mathrm{~d}$ & $2.70 \mathrm{c}$ & $1.70 \mathrm{~b}$ & $3.00 \mathrm{c}$ & $2.95 \mathrm{~b}$ & $12.72 \mathrm{c}$ \\
\hline TSxPT 184 & $2.01 \mathrm{~d}$ & $2.77 \mathrm{c}$ & $1.97 \mathrm{~b}$ & $3.03 \mathrm{c}$ & $4.18 \mathrm{~b}$ & $13.30 \mathrm{c}$ \\
\hline TSxPT 190 & $1.59 \mathrm{c}$ & $2.50 \mathrm{c}$ & $1.44 \mathrm{a}$ & $2.40 \mathrm{~b}$ & $1.74 \mathrm{a}$ & $7.50 \mathrm{~b}$ \\
\hline TSxPT 196 & $2.13 \mathrm{~d}$ & $2.87 \mathrm{~d}$ & $1.99 \mathrm{~b}$ & $2.90 \mathrm{c}$ & $4.39 \mathrm{~b}$ & $12.62 \mathrm{c}$ \\
\hline TSxPT 245 & $2.04 \mathrm{~d}$ & $2.63 \mathrm{c}$ & $1.53 \mathrm{a}$ & $2.93 \mathrm{c}$ & $2.51 \mathrm{a}$ & $11.88 \mathrm{c}$ \\
\hline TSxPT 248 & $2.12 \mathrm{~d}$ & $2.93 \mathrm{~d}$ & $1.80 \mathrm{~b}$ & $3.03 \mathrm{c}$ & $3.58 \mathrm{~b}$ & $14.23 \mathrm{c}$ \\
\hline TSxPT 254 & $2.20 \mathrm{~d}$ & $2.87 \mathrm{~d}$ & $2.08 \mathrm{~b}$ & $3.13 \mathrm{c}$ & $4.98 \mathrm{~b}$ & $14.75 \mathrm{~d}$ \\
\hline TSxPT 299 & $2.31 \mathrm{~d}$ & $2.50 \mathrm{c}$ & $1.76 \mathrm{~b}$ & $2.77 \mathrm{c}$ & $3.80 \mathrm{~b}$ & $9.95 \mathrm{~b}$ \\
\hline CV (\%) & 4.75 & 5.75 & 4.62 & 6.93 & 9.40 & 16.68 \\
\hline
\end{tabular}

Means followed by same letter do not differ by Scott-Knott test $(P \leq 0.05)$. 
There were differences in the yield of the plants in both years of evaluation. The highest fruit yield of Pera sweet orange were observed in plants grafted on hybrid TSxPT 155, 142, 54, 92, which had the cumulative production above $135 \mathrm{~kg}^{-1} \mathrm{tree}^{-1}$ (Table 2). Cristofani-Yaly et al. (2007) also observed that the hybrid TSxPT 54 was one of the most productive. The correlation between the volume of plant canopy and fruit production in 2010 was 0.68 ( $P$ $<0.05$ ) (Figure 2), showing that the most productive plants also showed higher canopy volumes. Plant yield was calculated in $\mathrm{kg}$ of fruit per cubic meter of canopy and an average of two years of evaluation, the values ranged from 5.0 to $12.4 \mathrm{~kg} \mathrm{~m}^{-3}$ (Table 2).

Table 2. Production, productivity and drought resistance of rootstock hybrids Sunki mandarin x Poncirus trifoliata grafted with Pera sweet orange. Colômbia, SP, Brazil, 2007-2010

\begin{tabular}{|c|c|c|c|c|c|c|c|c|c|}
\hline \multirow{2}{*}{ Hybrids } & \multicolumn{3}{|c|}{ Production $\left(\mathrm{kg}\right.$ tree $\left.{ }^{-1}\right)$} & \multicolumn{3}{|c|}{ Productivity $\left(\mathrm{kg} \mathrm{m}^{-5}\right)$} & \multicolumn{3}{|c|}{ Drought resistance } \\
\hline & 2007 & 2010 & Total & 2007 & 2010 & Mean & 2007 & 2010 & Mean \\
\hline TSxPT 7 & $25.3 \mathrm{a}$ & $42.7 \mathrm{~b}$ & $68.0 \mathrm{~b}$ & $10.0 \mathrm{a}$ & $4.7 \mathrm{a}$ & $7.4 \mathrm{a}$ & $1.7 \mathrm{a}$ & $2.0 \mathrm{~b}$ & $1.8 \mathrm{~b}$ \\
\hline TSxPT 14 & $15.8 \mathrm{a}$ & $10.7 \mathrm{a}$ & $26.5 \mathrm{a}$ & $18.2 \mathrm{~b}$ & $3.8 \mathrm{a}$ & $11.0 \mathrm{~b}$ & $1.0 \mathrm{a}$ & $1.0 \mathrm{a}$ & $1.0 \mathrm{a}$ \\
\hline TSxPT 16 & $32.0 \mathrm{~b}$ & $51.8 \mathrm{c}$ & $83.8 \mathrm{c}$ & $16.3 \mathrm{~b}$ & $7.4 \mathrm{a}$ & $11.8 \mathrm{~b}$ & $2.5 \mathrm{~b}$ & $2.0 \mathrm{~b}$ & $2.3 \mathrm{c}$ \\
\hline TSxPT 17 & $36.0 \mathrm{~b}$ & $57.5 \mathrm{c}$ & $93.5 \mathrm{c}$ & $12.8 \mathrm{~b}$ & $5.4 \mathrm{a}$ & $9.1 \mathrm{~b}$ & $2.0 \mathrm{a}$ & $1.3 \mathrm{a}$ & $1.7 \mathrm{~b}$ \\
\hline TSxPT 23 & $25.3 \mathrm{a}$ & $49.7 \mathrm{c}$ & $75.0 \mathrm{~b}$ & $13.7 \mathrm{~b}$ & $4.5 \mathrm{a}$ & $9.1 \mathrm{~b}$ & $1.3 \mathrm{a}$ & $1.7 \mathrm{a}$ & $1.5 \mathrm{~b}$ \\
\hline TSxPT 26 & $17.2 \mathrm{a}$ & $24.0 \mathrm{~b}$ & $41.2 \mathrm{a}$ & $13.9 \mathrm{~b}$ & $6.6 \mathrm{a}$ & $10.3 \mathrm{~b}$ & $1.0 \mathrm{a}$ & $1.0 \mathrm{a}$ & $1.0 \mathrm{a}$ \\
\hline TSxPT 38 & $17.1 \mathrm{a}$ & $27.6 \mathrm{~b}$ & $44.7 \mathrm{a}$ & $7.2 \mathrm{a}$ & $2.8 \mathrm{a}$ & $5.0 \mathrm{a}$ & $1.5 \mathrm{a}$ & $1.0 \mathrm{a}$ & $1.3 \mathrm{a}$ \\
\hline TSxPT 42 & $27.0 \mathrm{a}$ & $27.6 \mathrm{~b}$ & $54.6 \mathrm{~b}$ & $10.4 \mathrm{a}$ & $2.5 \mathrm{a}$ & $6.5 \mathrm{a}$ & $2.0 \mathrm{a}$ & $2.0 \mathrm{~b}$ & $2.0 \mathrm{~b}$ \\
\hline TSxPT 54 & $66.0 \mathrm{~b}$ & $77.7 \mathrm{c}$ & $143.7 \mathrm{c}$ & $13.0 \mathrm{~b}$ & $5.3 \mathrm{a}$ & $9.1 \mathrm{~b}$ & $3.0 \mathrm{~b}$ & $1.7 \mathrm{a}$ & $2.3 \mathrm{c}$ \\
\hline TSxPT 56 & $27.3 \mathrm{a}$ & $39.0 \mathrm{~b}$ & $66.4 \mathrm{~b}$ & $11.9 \mathrm{a}$ & $4.5 \mathrm{a}$ & $8.2 \mathrm{a}$ & $2.0 \mathrm{a}$ & $1.3 \mathrm{a}$ & $1.7 \mathrm{~b}$ \\
\hline TSxPT 68 & $18.7 \mathrm{a}$ & $30.3 \mathrm{~b}$ & $48.9 \mathrm{~b}$ & $19.7 \mathrm{~b}$ & $4.8 \mathrm{a}$ & $12.2 \mathrm{~b}$ & $2.7 \mathrm{~b}$ & $1.0 \mathrm{a}$ & $1.8 \mathrm{~b}$ \\
\hline TSxPT 70 & $35.8 \mathrm{~b}$ & $39.0 \mathrm{~b}$ & $74.8 \mathrm{~b}$ & $17.2 \mathrm{~b}$ & $7.7 \mathrm{a}$ & $12.4 \mathrm{~b}$ & $3.0 \mathrm{~b}$ & $2.3 \mathrm{~b}$ & $2.7 \mathrm{c}$ \\
\hline TSxPT 86 & $42.0 \mathrm{~b}$ & $41.2 \mathrm{~b}$ & $83.2 \mathrm{c}$ & $8.6 \mathrm{a}$ & $3.4 \mathrm{a}$ & $6.0 \mathrm{a}$ & $2.5 \mathrm{~b}$ & $2.0 \mathrm{~b}$ & $2.3 \mathrm{c}$ \\
\hline TSxPT 92 & $52.7 \mathrm{~b}$ & $82.4 \mathrm{c}$ & $135.1 \mathrm{c}$ & $14.4 \mathrm{~b}$ & $4.5 \mathrm{a}$ & $9.5 \mathrm{~b}$ & $3.0 \mathrm{~b}$ & $2.3 \mathrm{~b}$ & $2.7 \mathrm{c}$ \\
\hline TSxPT 101 & $33.4 \mathrm{~b}$ & $68.7 \mathrm{c}$ & $102.2 \mathrm{c}$ & $12.0 \mathrm{a}$ & $5.3 \mathrm{a}$ & $8.6 \mathrm{~b}$ & $2.7 \mathrm{~b}$ & $2.0 \mathrm{~b}$ & $2.3 \mathrm{c}$ \\
\hline TSxPT 106 & $39.3 \mathrm{~b}$ & $69.5 \mathrm{c}$ & $108.8 \mathrm{c}$ & $11.4 \mathrm{a}$ & $7.0 \mathrm{a}$ & $9.2 \mathrm{~b}$ & $2.3 \mathrm{~b}$ & $1.7 \mathrm{a}$ & $2.0 \mathrm{~b}$ \\
\hline TSxPT 107 & $25.4 \mathrm{a}$ & $35.8 \mathrm{~b}$ & $62.2 \mathrm{~b}$ & $10.2 \mathrm{a}$ & $3.6 \mathrm{a}$ & $6.9 \mathrm{a}$ & $1.3 \mathrm{a}$ & $2.0 \mathrm{~b}$ & $1.7 \mathrm{~b}$ \\
\hline TSxPT 108 & $45.9 \mathrm{~b}$ & $72.3 \mathrm{c}$ & $118.2 \mathrm{c}$ & $13.2 \mathrm{~b}$ & $4.7 \mathrm{a}$ & $8.9 \mathrm{~b}$ & $3.0 \mathrm{~b}$ & $2.0 \mathrm{~b}$ & $2.5 \mathrm{c}$ \\
\hline TSxPT 110 & $14.6 \mathrm{a}$ & $9.8 \mathrm{a}$ & $24.4 \mathrm{a}$ & $16.2 \mathrm{~b}$ & $3.2 \mathrm{a}$ & $9.7 \mathrm{~b}$ & $1.3 \mathrm{a}$ & $1.3 \mathrm{a}$ & $1.3 \mathrm{a}$ \\
\hline TSxPT 119 & $28.3 \mathrm{a}$ & $76.9 \mathrm{c}$ & $105.2 \mathrm{c}$ & $11.0 \mathrm{a}$ & $5.5 \mathrm{a}$ & $8.3 \mathrm{~b}$ & $3.0 \mathrm{~b}$ & $2.0 \mathrm{~b}$ & $2.5 \mathrm{c}$ \\
\hline TSxPT 121 & $16.5 \mathrm{a}$ & $22.8 \mathrm{~b}$ & $39.3 \mathrm{a}$ & $9.6 \mathrm{a}$ & $2.6 \mathrm{a}$ & $6.1 \mathrm{a}$ & $1.7 \mathrm{a}$ & $1.0 \mathrm{a}$ & $1.3 \mathrm{a}$ \\
\hline TSxPT 124 & $43.3 \mathrm{~b}$ & $78.6 \mathrm{c}$ & $121.9 \mathrm{c}$ & $11.0 \mathrm{a}$ & $7.3 \mathrm{a}$ & $9.2 \mathrm{~b}$ & $2.3 \mathrm{~b}$ & $2.7 \mathrm{~b}$ & $2.5 \mathrm{c}$ \\
\hline TSxPT 126 & $35.0 \mathrm{~b}$ & $66.7 \mathrm{c}$ & $101.7 \mathrm{c}$ & $11.3 \mathrm{a}$ & $4.4 \mathrm{a}$ & $7.9 \mathrm{a}$ & $3.0 \mathrm{~b}$ & $2.0 \mathrm{~b}$ & $2.5 \mathrm{c}$ \\
\hline TSxPT 128 & $32.3 \mathrm{~b}$ & $71.0 \mathrm{c}$ & $103.3 \mathrm{c}$ & $12.8 \mathrm{~b}$ & $7.2 \mathrm{a}$ & $10.0 \mathrm{~b}$ & $3.0 \mathrm{~b}$ & $2.7 \mathrm{~b}$ & $2.8 \mathrm{c}$ \\
\hline TSxPT 132 & $30.4 \mathrm{a}$ & $90.7 \mathrm{c}$ & $121.1 \mathrm{c}$ & $8.0 \mathrm{a}$ & $5.2 \mathrm{a}$ & $6.6 \mathrm{a}$ & $2.7 \mathrm{~b}$ & $3.0 \mathrm{~b}$ & $2.8 \mathrm{c}$ \\
\hline TSxPT 136 & $14.4 \mathrm{a}$ & $24.6 \mathrm{~b}$ & $38.9 \mathrm{a}$ & $12.2 \mathrm{a}$ & $4.1 \mathrm{a}$ & $8.1 \mathrm{a}$ & $1.7 \mathrm{a}$ & $1.0 \mathrm{a}$ & $1.3 \mathrm{a}$ \\
\hline TSxPT 137 & $12.8 \mathrm{a}$ & $16.0 \mathrm{a}$ & $28.8 \mathrm{a}$ & $10.7 \mathrm{a}$ & $3.2 \mathrm{a}$ & $7.0 \mathrm{a}$ & $1.0 \mathrm{a}$ & $1.0 \mathrm{a}$ & $1.0 \mathrm{a}$ \\
\hline TSxPT 139 & $37.2 \mathrm{~b}$ & $73.7 \mathrm{c}$ & $110.9 \mathrm{c}$ & $13.3 \mathrm{~b}$ & $7.2 \mathrm{a}$ & $10.3 \mathrm{~b}$ & $1.7 \mathrm{a}$ & $2.0 \mathrm{~b}$ & $1.8 \mathrm{~b}$ \\
\hline TSxPT 142 & $50.3 \mathrm{~b}$ & $95.2 \mathrm{c}$ & $145.4 \mathrm{c}$ & $17.3 \mathrm{~b}$ & $5.5 \mathrm{a}$ & $11.4 \mathrm{~b}$ & $3.0 \mathrm{~b}$ & $3.0 \mathrm{~b}$ & $3.0 \mathrm{c}$ \\
\hline TSxPT 143 & $29.5 \mathrm{a}$ & $37.9 \mathrm{~b}$ & $67.4 \mathrm{~b}$ & $9.7 \mathrm{a}$ & $4.8 \mathrm{a}$ & $7.3 \mathrm{a}$ & $2.0 \mathrm{a}$ & $1.7 \mathrm{a}$ & $1.8 \mathrm{~b}$ \\
\hline TSxPT 148 & $19.8 \mathrm{a}$ & $37.4 \mathrm{~b}$ & $57.2 \mathrm{~b}$ & $7.4 \mathrm{a}$ & $4.2 \mathrm{a}$ & $5.8 \mathrm{a}$ & $1.7 \mathrm{a}$ & $1.0 \mathrm{a}$ & $1.3 \mathrm{a}$ \\
\hline TSxPT 152 & $24.9 \mathrm{a}$ & $78.6 \mathrm{c}$ & $103.5 \mathrm{c}$ & $9.3 \mathrm{a}$ & $6.1 \mathrm{a}$ & $7.7 \mathrm{a}$ & $2.7 \mathrm{~b}$ & $2.7 \mathrm{~b}$ & $2.7 \mathrm{c}$ \\
\hline TSxPT 155 & $52.5 \mathrm{~b}$ & $119.6 \mathrm{c}$ & $172.1 \mathrm{c}$ & $11.2 \mathrm{a}$ & $6.3 \mathrm{a}$ & $8.7 \mathrm{~b}$ & $3.0 \mathrm{~b}$ & $2.7 \mathrm{~b}$ & $2.8 \mathrm{c}$ \\
\hline TSxPT 166 & $30.0 \mathrm{a}$ & $60.0 \mathrm{c}$ & $90.0 \mathrm{c}$ & $8.1 \mathrm{a}$ & $5.3 \mathrm{a}$ & $6.7 \mathrm{a}$ & $2.7 \mathrm{~b}$ & $2.3 \mathrm{~b}$ & $2.5 \mathrm{c}$ \\
\hline TSxPT 168 & $26.3 \mathrm{a}$ & $58.5 \mathrm{c}$ & $84.8 \mathrm{~b}$ & $9.0 \mathrm{a}$ & $4.7 \mathrm{a}$ & $6.9 \mathrm{a}$ & $3.0 \mathrm{~b}$ & $2.3 \mathrm{~b}$ & $2.7 \mathrm{c}$ \\
\hline TSxPT 184 & $42.7 \mathrm{~b}$ & $71.0 \mathrm{c}$ & $113.8 \mathrm{c}$ & $10.5 \mathrm{a}$ & $5.3 \mathrm{a}$ & $7.9 \mathrm{a}$ & $2.7 \mathrm{~b}$ & $2.3 \mathrm{~b}$ & $2.5 \mathrm{c}$ \\
\hline TSxPT 190 & $29.2 \mathrm{a}$ & $46.9 \mathrm{~b}$ & $76.1 \mathrm{~b}$ & $16.8 \mathrm{~b}$ & $6.2 \mathrm{a}$ & $11.5 \mathrm{~b}$ & $2.3 \mathrm{~b}$ & $1.7 \mathrm{a}$ & $2.0 \mathrm{~b}$ \\
\hline TSxPT 196 & $36.6 \mathrm{~b}$ & $61.2 \mathrm{c}$ & $97.8 \mathrm{c}$ & $8.4 \mathrm{a}$ & $4.8 \mathrm{a}$ & $6.6 \mathrm{a}$ & $2.7 \mathrm{~b}$ & $1.7 \mathrm{a}$ & $2.2 \mathrm{c}$ \\
\hline TSxPT 245 & $38.2 \mathrm{~b}$ & $62.9 \mathrm{c}$ & $101.1 \mathrm{c}$ & $15.4 \mathrm{~b}$ & $5.4 \mathrm{a}$ & $10.4 \mathrm{~b}$ & $3.0 \mathrm{~b}$ & $2.0 \mathrm{~b}$ & $2.5 \mathrm{c}$ \\
\hline TSxPT 248 & $29.3 \mathrm{a}$ & $66.6 \mathrm{c}$ & $95.9 \mathrm{c}$ & $8.1 \mathrm{a}$ & $4.8 \mathrm{a}$ & $6.5 \mathrm{a}$ & $2.3 \mathrm{~b}$ & $3.0 \mathrm{~b}$ & $2.7 \mathrm{c}$ \\
\hline TSxPT 254 & $37.1 \mathrm{~b}$ & $70.8 \mathrm{c}$ & $107.9 \mathrm{c}$ & $7.5 \mathrm{a}$ & $4.9 \mathrm{a}$ & $6.1 \mathrm{a}$ & $2.7 \mathrm{~b}$ & $2.7 \mathrm{~b}$ & $2.7 \mathrm{c}$ \\
\hline TSxPT 299 & $35.4 \mathrm{~b}$ & $84.0 \mathrm{c}$ & $119.4 \mathrm{c}$ & $9.5 \mathrm{a}$ & $8.4 \mathrm{a}$ & $8.9 \mathrm{~b}$ & $3.0 \mathrm{~b}$ & $3.0 \mathrm{~b}$ & $3.0 \mathrm{c}$ \\
\hline CV (\%) & 20.5 & 18.0 & & 19.9 & 19.6 & & 13.3 & 14.5 & \\
\hline
\end{tabular}

Means followed by same letter do not differ by Scott-Knott test $(P \leq 0.05)$. 
Significant differences were observed for the variables mass $(\mathrm{g})$, height $(\mathrm{cm})$ and diameter $(\mathrm{cm})$ of fruit, juice yield (\%) and number of fruits per $40.8 \mathrm{~kg}$ box (Table 3). For the height/diameter of fruits no differences were observed. For fruit mass values were between $180 \mathrm{~g}$ (TSxPT 166) and $289 \mathrm{~g}$ (TSxPT 121). For juice yield values were between $42 \%$ (TSxPT 38) and 56\% (TSxPT 299).

Table 3. Physical characteristics of the fruits of Pera sweet orange grafted on hybrids of Sunki mandarin $x$ Poncirus trifoliata. Colômbia, São Paulo, Brazil, 2009

\begin{tabular}{|c|c|c|c|c|c|c|}
\hline Hybrids & $\begin{array}{l}\text { Mass } \\
(\mathrm{g})\end{array}$ & $\begin{array}{l}\text { Height } \\
(\mathrm{cm})\end{array}$ & $\begin{array}{l}\text { Diameter } \\
(\mathrm{cm})\end{array}$ & $\begin{array}{l}\text { Ratio } \\
\text { H/D }\end{array}$ & $\begin{array}{l}\text { Juice yield } \\
(\%)\end{array}$ & $\begin{array}{l}\mathrm{N}^{0} \text { Fruits } \\
\text { box }^{-1} *\end{array}$ \\
\hline TSxPT 7 & $206.3 \mathrm{a}$ & $7.53 \mathrm{a}$ & $7.53 \mathrm{~b}$ & $1.00 \mathrm{a}$ & $48.2 \mathrm{~b}$ & $203 \mathrm{~b}$ \\
\hline TSxPT 14 & $208.0 \mathrm{a}$ & $7.80 \mathrm{a}$ & $7.30 \mathrm{a}$ & $1.07 \mathrm{a}$ & $47.1 \mathrm{~b}$ & $196 \mathrm{~b}$ \\
\hline TSxPT 16 & $242.5 b$ & $8.10 \mathrm{~b}$ & $7.70 \mathrm{~b}$ & $1.05 \mathrm{a}$ & $51.2 \mathrm{c}$ & $169 \mathrm{a}$ \\
\hline TSxPT 17 & $227.7 \mathrm{a}$ & $7.97 \mathrm{~b}$ & $7.37 \mathrm{a}$ & $1.08 \mathrm{a}$ & $47.4 \mathrm{~b}$ & $180 \mathrm{a}$ \\
\hline TSxPT 23 & $230.3 \mathrm{a}$ & $8.03 \mathrm{~b}$ & $7.60 \mathrm{~b}$ & $1.06 \mathrm{a}$ & $51.3 \mathrm{c}$ & $179 \mathrm{a}$ \\
\hline TSxPT 26 & $245.0 \mathrm{~b}$ & $8.20 \mathrm{~b}$ & $7.60 \mathrm{~b}$ & $1.08 \mathrm{a}$ & $48.5 \mathrm{~b}$ & $167 \mathrm{a}$ \\
\hline TSxPT 38 & $227.0 \mathrm{a}$ & $8.00 \mathrm{~b}$ & $7.65 \mathrm{~b}$ & $1.05 \mathrm{a}$ & $41.9 \mathrm{a}$ & $180 \mathrm{a}$ \\
\hline TSxPT 42 & $203.0 \mathrm{a}$ & $7.60 \mathrm{a}$ & $7.30 \mathrm{a}$ & $1.04 \mathrm{a}$ & $53.2 \mathrm{~d}$ & $201 \mathrm{~b}$ \\
\hline TSxPT 54 & $224.5 \mathrm{a}$ & $8.00 \mathrm{~b}$ & $7.35 \mathrm{a}$ & $1.09 \mathrm{a}$ & $49.8 \mathrm{c}$ & $185 \mathrm{~b}$ \\
\hline TSxPT 56 & $217.7 \mathrm{a}$ & $7.93 \mathrm{a}$ & $7.37 \mathrm{a}$ & $1.08 \mathrm{a}$ & $47.9 \mathrm{~b}$ & $190 \mathrm{~b}$ \\
\hline TSxPT 68 & $207.5 \mathrm{a}$ & $7.75 \mathrm{a}$ & $7.10 \mathrm{a}$ & $1.09 \mathrm{a}$ & $48.5 \mathrm{~b}$ & $201 \mathrm{~b}$ \\
\hline TSxPT 70 & $198.0 \mathrm{a}$ & $7.63 \mathrm{a}$ & $7.20 \mathrm{a}$ & $1.06 \mathrm{a}$ & $51.1 \mathrm{c}$ & $208 \mathrm{~b}$ \\
\hline TSxPT 86 & $196.0 \mathrm{a}$ & $7.55 \mathrm{a}$ & $7.15 \mathrm{a}$ & $1.06 \mathrm{a}$ & $51.8 \mathrm{c}$ & $209 \mathrm{~b}$ \\
\hline TSxPT 92 & $250.0 \mathrm{~b}$ & $8.43 \mathrm{~b}$ & $7.57 \mathrm{~b}$ & $1.12 \mathrm{a}$ & $48.4 \mathrm{~b}$ & $164 \mathrm{a}$ \\
\hline TSxPT 101 & $244.2 \mathrm{~b}$ & $8.20 \mathrm{~b}$ & $7.70 \mathrm{~b}$ & $1.07 \mathrm{a}$ & $54.3 \mathrm{~d}$ & $167 \mathrm{a}$ \\
\hline TSxPT 106 & $208.5 \mathrm{a}$ & $7.70 \mathrm{a}$ & $7.30 \mathrm{a}$ & $1.06 \mathrm{a}$ & $50.9 \mathrm{c}$ & $196 \mathrm{~b}$ \\
\hline TSxPT 107 & $201.0 \mathrm{a}$ & $7.70 \mathrm{a}$ & $7.10 \mathrm{a}$ & $1.09 \mathrm{a}$ & $43.6 \mathrm{a}$ & $206 \mathrm{~b}$ \\
\hline TSxPT 108 & $210.0 \mathrm{a}$ & $7.70 \mathrm{a}$ & $7.30 \mathrm{a}$ & $1.05 \mathrm{a}$ & $50.9 \mathrm{c}$ & $199 \mathrm{~b}$ \\
\hline TSxPT 110 & $266.0 \mathrm{~b}$ & $8.65 \mathrm{~b}$ & $7.90 \mathrm{~b}$ & $1.10 \mathrm{a}$ & $44.2 \mathrm{a}$ & $155 \mathrm{a}$ \\
\hline TSxPT 119 & $243.0 \mathrm{~b}$ & $8.15 \mathrm{~b}$ & $7.55 \mathrm{~b}$ & $1.08 \mathrm{a}$ & $50.5 \mathrm{c}$ & $168 \mathrm{a}$ \\
\hline TSxPT 121 & $288.5 \mathrm{~b}$ & $8.70 \mathrm{~b}$ & $7.45 \mathrm{~b}$ & $1.17 \mathrm{a}$ & $48.0 \mathrm{~b}$ & $143 \mathrm{a}$ \\
\hline TSxPT 124 & $221.3 \mathrm{a}$ & $7.87 \mathrm{a}$ & $7.40 \mathrm{a}$ & $1.06 \mathrm{a}$ & $51.3 \mathrm{c}$ & $185 \mathrm{~b}$ \\
\hline TSxPT 126 & $250.0 \mathrm{~b}$ & $8.33 \mathrm{~b}$ & $7.67 \mathrm{~b}$ & $1.09 \mathrm{a}$ & $48.9 \mathrm{~b}$ & $165 \mathrm{a}$ \\
\hline TSxPT 128 & $208.7 \mathrm{a}$ & $7.67 \mathrm{a}$ & $7.10 \mathrm{a}$ & $1.08 \mathrm{a}$ & $51.2 \mathrm{c}$ & $197 \mathrm{~b}$ \\
\hline TSxPT 132 & $237.7 \mathrm{~b}$ & $8.10 \mathrm{~b}$ & $7.60 \mathrm{~b}$ & $1.07 \mathrm{a}$ & $53.1 \mathrm{~d}$ & $172 \mathrm{a}$ \\
\hline TSxPT 136 & $228.0 \mathrm{a}$ & $8.00 \mathrm{~b}$ & $7.60 \mathrm{~b}$ & $1.05 \mathrm{a}$ & $49.1 \mathrm{~b}$ & $179 \mathrm{a}$ \\
\hline TSxPT 137 & $212.7 \mathrm{a}$ & $7.67 \mathrm{a}$ & $7.37 \mathrm{a}$ & $1.04 \mathrm{a}$ & $46.8 \mathrm{~b}$ & $193 \mathrm{~b}$ \\
\hline TSxPT 139 & $225.0 \mathrm{a}$ & $8.03 \mathrm{~b}$ & $7.43 \mathrm{~b}$ & $1.08 \mathrm{a}$ & $50.3 \mathrm{c}$ & $186 \mathrm{~b}$ \\
\hline TSxPT 142 & $227.0 \mathrm{a}$ & $8.03 \mathrm{~b}$ & $7.27 \mathrm{a}$ & $1.11 \mathrm{a}$ & $49.5 \mathrm{c}$ & $180 \mathrm{a}$ \\
\hline TSxPT 143 & $202.5 \mathrm{a}$ & $7.60 \mathrm{a}$ & $7.55 \mathrm{~b}$ & $1.01 \mathrm{a}$ & $49.4 \mathrm{c}$ & $204 \mathrm{~b}$ \\
\hline TSxPT 148 & $234.3 \mathrm{~b}$ & $8.10 \mathrm{~b}$ & $7.60 \mathrm{~b}$ & $1.07 \mathrm{a}$ & $46.8 \mathrm{~b}$ & $175 \mathrm{a}$ \\
\hline TSxPT 152 & $218.3 \mathrm{a}$ & $7.90 \mathrm{a}$ & $7.33 \mathrm{a}$ & $1.08 \mathrm{a}$ & $51.3 \mathrm{c}$ & $187 \mathrm{~b}$ \\
\hline TSxPT 155 & $221.3 \mathrm{a}$ & $8.20 \mathrm{~b}$ & $7.47 \mathrm{~b}$ & $1.10 \mathrm{a}$ & $48.6 \mathrm{~b}$ & $185 \mathrm{~b}$ \\
\hline TSxPT 166 & $180.3 \mathrm{a}$ & $7.33 \mathrm{a}$ & $6.97 \mathrm{a}$ & $1.05 \mathrm{a}$ & $50.4 \mathrm{c}$ & $229 \mathrm{~b}$ \\
\hline TSxPT 168 & $233.0 \mathrm{~b}$ & $8.13 \mathrm{~b}$ & $7.50 \mathrm{~b}$ & $1.09 \mathrm{a}$ & $51.0 \mathrm{c}$ & $177 \mathrm{a}$ \\
\hline TSxPT 184 & $216.7 \mathrm{a}$ & $7.73 \mathrm{a}$ & $7.33 \mathrm{a}$ & $1.06 \mathrm{a}$ & $48.8 \mathrm{~b}$ & $190 \mathrm{~b}$ \\
\hline TSxPT 190 & $257.3 \mathrm{~b}$ & $8.40 \mathrm{~b}$ & $7.73 \mathrm{~b}$ & $1.09 \mathrm{a}$ & $50.1 \mathrm{c}$ & $161 \mathrm{a}$ \\
\hline TSxPT 196 & $219.0 \mathrm{a}$ & $7.90 \mathrm{a}$ & $7.57 \mathrm{~b}$ & $1.04 \mathrm{a}$ & $51.7 \mathrm{c}$ & $187 \mathrm{~b}$ \\
\hline TSxPT 245 & $218.3 \mathrm{a}$ & $8.00 \mathrm{~b}$ & $7.27 \mathrm{a}$ & $1.10 \mathrm{a}$ & $49.9 \mathrm{c}$ & $187 \mathrm{~b}$ \\
\hline TSxPT 248 & $208.0 \mathrm{a}$ & $7.90 \mathrm{a}$ & $7.33 \mathrm{a}$ & $1.08 \mathrm{a}$ & $51.7 \mathrm{c}$ & $197 \mathrm{~b}$ \\
\hline TSxPT 254 & $203.0 \mathrm{a}$ & $7.70 \mathrm{a}$ & $7.20 \mathrm{a}$ & $1.07 \mathrm{a}$ & $50.6 \mathrm{c}$ & $202 \mathrm{~b}$ \\
\hline TSxPT 299 & $211.3 \mathrm{a}$ & $7.80 \mathrm{a}$ & $7.20 \mathrm{a}$ & $1.08 \mathrm{a}$ & $56.4 \mathrm{~d}$ & $194 \mathrm{~b}$ \\
\hline CV (\%) & 10.06 & 3.95 & 3.31 & 2.98 & 4.08 & 10.21 \\
\hline
\end{tabular}

Means followed by same letter do not differ by Scott-Knott test $(P \leq 0.05)$.

*Box of $40.8 \mathrm{~kg}$ of fruits. 


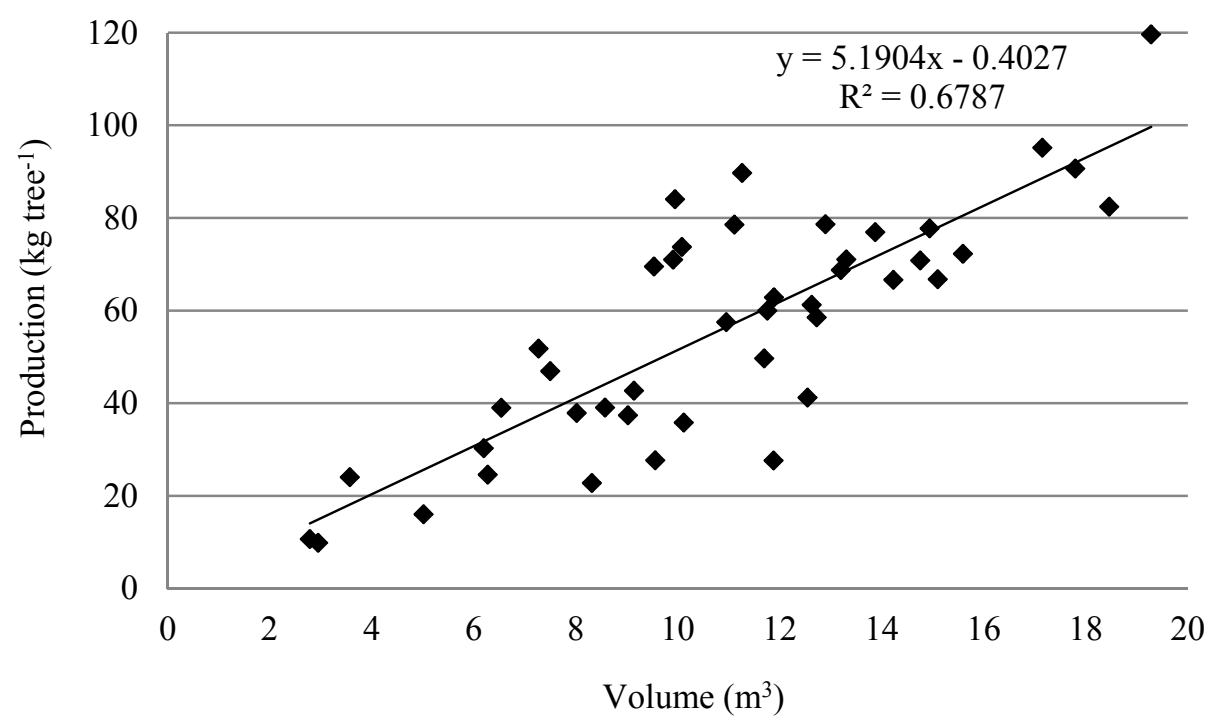

Figure 2. Correlation between production $\left(\mathrm{kg}\right.$ tree $\left.\mathrm{e}^{-1}\right)$ and canopy volume $\left(\mathrm{m}^{3}\right)$ of Pera sweet orange plants grafted on 42 hybrids of Sunki mandarin x Poncirus trifoliata (Colômbia, SP, Brazil, 2010),

We observed significant differences among the rootstocks for acidity, Brix, ratio and total soluble solids per box with $40.8 \mathrm{~kg}$ of fruit (Table 4). Di Giorgi et al. (1994) report that for Pera sweet orange, the fruit must be harvested when normally meet acidity levels between 0.6 to $0.9 \%$, achieving this stage largest concentrations of soluble solids and lower rates of degradation of fruit that causes the loss of their commercial value both for fresh fruit market or for processing in the industry. Thus, it was found that during assessment of internal qualities of the fruit (October/2009), the juice acidity ranged from 0.58 (TSxPT 121) to 1.29 (TSxPT 128), and most part of the canopy / rootstock combinations studied were within the standards set for harvest (Table 4).

For the values of ratio, Viégas (1991) reports that the range between 11 and 14 is ideal for industrialization and that these levels the acidity are still adequate to maintain the quality and conservation of juice after processing. In this study the majority of canopy / rootstock combination showed ratio values within the ideal range, however, some hybrids TSxPT $(42,121,142,152,190$ and 248) induced early ripening of the fruits of Pera sweet orange, with values above 15.5. Moreover, the hybrids TSxPT 68,110, 128, 136 and 143 had values of ratio below 10, providing late maturation of the fruits of Pera sweet orange (Table 4). 
Table 4. Chemical characteristics of the fruits of Pera sweet orange grafted on hybrids of Sunki mandarin $\mathrm{x}$ Poncirus trifoliata. Colômbia, São Paulo, Brazil, 2009

\begin{tabular}{|c|c|c|c|c|}
\hline Hybrids & $\begin{array}{l}\text { Acidity } \\
\left(\mathrm{g} 100 \mathrm{~mL}^{-1}\right)\end{array}$ & ${ }^{\circ}$ Brix & Ratio & $\begin{array}{l}\text { TSS } \\
\left(\operatorname{kg~box}^{-1}\right)\end{array}$ \\
\hline TSxPT 7 & $0.901 \mathrm{~b}$ & $11.3 \mathrm{~b}$ & $14.0 \mathrm{~b}$ & $2.24 \mathrm{~b}$ \\
\hline TSxPT 14 & $0.883 \mathrm{~b}$ & $9.9 \mathrm{a}$ & $11.2 \mathrm{a}$ & $1.90 \mathrm{a}$ \\
\hline TSxPT 16 & $0.779 \mathrm{a}$ & $10.6 \mathrm{~b}$ & $13.5 \mathrm{~b}$ & $2.19 \mathrm{~b}$ \\
\hline TSxPT 17 & $0.992 \mathrm{~b}$ & $9.8 \mathrm{a}$ & $10.2 \mathrm{a}$ & $1.90 \mathrm{a}$ \\
\hline TSxPT 23 & $0.851 \mathrm{a}$ & $9.2 \mathrm{a}$ & $11.7 \mathrm{a}$ & $1.92 \mathrm{a}$ \\
\hline TSxPT 26 & $0.973 \mathrm{~b}$ & $10.0 \mathrm{a}$ & $11.4 \mathrm{a}$ & $1.98 \mathrm{a}$ \\
\hline TSxPT 38 & $1.084 \mathrm{~b}$ & $10.7 \mathrm{~b}$ & $10.2 \mathrm{a}$ & $1.82 \mathrm{a}$ \\
\hline TSxPT 42 & $0.619 \mathrm{a}$ & $10.5 \mathrm{~b}$ & $17.0 \mathrm{~b}$ & $2.28 \mathrm{~b}$ \\
\hline TSxPT 54 & $0.711 \mathrm{a}$ & $10.6 \mathrm{~b}$ & $15.1 \mathrm{~b}$ & $2.15 \mathrm{~b}$ \\
\hline TSxPT 56 & $0.661 \mathrm{a}$ & $10.0 \mathrm{a}$ & $15.2 \mathrm{~b}$ & $1.97 \mathrm{a}$ \\
\hline TSxPT 68 & $1.167 \mathrm{~b}$ & $9.5 \mathrm{a}$ & $8.2 \mathrm{a}$ & $1.89 \mathrm{a}$ \\
\hline TSxPT 70 & $0.914 \mathrm{~b}$ & $11.0 \mathrm{~b}$ & $12.9 \mathrm{~b}$ & $2.29 \mathrm{~b}$ \\
\hline TSxPT 86 & $0.826 \mathrm{a}$ & $11.2 \mathrm{~b}$ & $13.6 \mathrm{~b}$ & $2.36 \mathrm{~b}$ \\
\hline TSxPT 92 & $0.749 \mathrm{a}$ & $10.1 \mathrm{a}$ & $13.6 \mathrm{~b}$ & $1.99 \mathrm{a}$ \\
\hline TSxPT 101 & $0.608 \mathrm{a}$ & $9.3 \mathrm{a}$ & $15.3 \mathrm{~b}$ & $2.06 \mathrm{a}$ \\
\hline TSxPT 106 & $0.992 \mathrm{~b}$ & $10.8 \mathrm{~b}$ & $11.0 \mathrm{a}$ & $2.23 \mathrm{~b}$ \\
\hline TSxPT 107 & $0.874 \mathrm{~b}$ & $9.0 \mathrm{a}$ & $10.3 \mathrm{a}$ & $1.61 \mathrm{a}$ \\
\hline TSxPT 108 & $0.764 \mathrm{a}$ & $11.1 \mathrm{~b}$ & $14.9 \mathrm{~b}$ & $2.31 \mathrm{~b}$ \\
\hline TSxPT 110 & $1.059 \mathrm{~b}$ & $10.0 \mathrm{a}$ & $9.5 \mathrm{a}$ & $1.81 \mathrm{a}$ \\
\hline TSxPT 119 & $0.723 \mathrm{a}$ & $9.8 \mathrm{a}$ & $13.5 \mathrm{~b}$ & $2.01 \mathrm{a}$ \\
\hline TSxPT 121 & $0.579 \mathrm{a}$ & $9.5 \mathrm{a}$ & $16.4 \mathrm{~b}$ & $1.87 \mathrm{a}$ \\
\hline TSxPT 124 & $0.672 \mathrm{a}$ & $10.0 \mathrm{a}$ & $14.9 \mathrm{~b}$ & $2.10 \mathrm{~b}$ \\
\hline TSxPT 126 & $0.704 \mathrm{a}$ & $9.4 \mathrm{a}$ & $13.6 \mathrm{~b}$ & $1.88 \mathrm{a}$ \\
\hline TSxPT 128 & $1.289 \mathrm{~b}$ & $10.5 \mathrm{~b}$ & $9.4 \mathrm{a}$ & $2.21 \mathrm{~b}$ \\
\hline TSxPT 132 & $0.745 \mathrm{a}$ & $10.9 \mathrm{~b}$ & $14.7 \mathrm{~b}$ & $2.36 \mathrm{~b}$ \\
\hline TSxPT 136 & $1.124 \mathrm{~b}$ & $9.9 \mathrm{a}$ & $8.8 \mathrm{a}$ & $1.99 \mathrm{a}$ \\
\hline TSxPT 137 & $0.875 \mathrm{~b}$ & $10.0 \mathrm{~b}$ & $12.8 \mathrm{~b}$ & $1.90 \mathrm{a}$ \\
\hline TSxPT 139 & $0.968 \mathrm{~b}$ & $9.9 \mathrm{a}$ & $11.4 \mathrm{a}$ & $2.02 \mathrm{a}$ \\
\hline TSxPT 142 & $0.711 \mathrm{a}$ & $11.1 \mathrm{~b}$ & $15.8 \mathrm{~b}$ & $2.25 \mathrm{~b}$ \\
\hline TSxPT 143 & $1.231 \mathrm{~b}$ & $11.1 \mathrm{~b}$ & $9.8 \mathrm{a}$ & $2.23 \mathrm{~b}$ \\
\hline TSxPT 148 & $0.639 \mathrm{a}$ & $9.6 \mathrm{a}$ & $15.4 \mathrm{~b}$ & $1.85 \mathrm{a}$ \\
\hline TSxPT 152 & $0.677 \mathrm{a}$ & $11.0 \mathrm{~b}$ & $16.6 \mathrm{~b}$ & $2.29 \mathrm{~b}$ \\
\hline TSxPT 155 & $0.764 \mathrm{a}$ & $9.8 \mathrm{a}$ & $13.3 \mathrm{~b}$ & $1.96 \mathrm{a}$ \\
\hline TSxPT 166 & $0.940 \mathrm{~b}$ & $10.3 \mathrm{a}$ & $12.1 \mathrm{a}$ & $2.12 \mathrm{~b}$ \\
\hline TSxPT 168 & $0.744 \mathrm{a}$ & $11.2 \mathrm{~b}$ & $15.1 \mathrm{~b}$ & $2.33 \mathrm{~b}$ \\
\hline TSxPT 184 & $0.870 \mathrm{~b}$ & $10.0 \mathrm{a}$ & $12.5 \mathrm{a}$ & $2.00 \mathrm{a}$ \\
\hline TSxPT 190 & $0.656 \mathrm{a}$ & $9.9 \mathrm{a}$ & $15.5 \mathrm{~b}$ & $2.02 \mathrm{a}$ \\
\hline TSxPT 196 & $0.774 \mathrm{a}$ & $11.7 \mathrm{~b}$ & $15.1 \mathrm{~b}$ & $2.46 \mathrm{~b}$ \\
\hline TSxPT 245 & $0.736 \mathrm{a}$ & $11.1 \mathrm{~b}$ & $15.2 \mathrm{~b}$ & $2.27 \mathrm{~b}$ \\
\hline TSxPT 248 & $0.635 \mathrm{a}$ & $10.1 \mathrm{a}$ & $16.6 \mathrm{~b}$ & $2.14 \mathrm{~b}$ \\
\hline TSxPT 254 & $0.744 \mathrm{a}$ & $11.1 \mathrm{~b}$ & $14.9 \mathrm{~b}$ & $2.28 \mathrm{~b}$ \\
\hline TSxPT 299 & $0.970 \mathrm{~b}$ & $10.4 \mathrm{a}$ & $11.7 \mathrm{a}$ & $2.38 \mathrm{~b}$ \\
\hline CV (\%) & 25.32 & 7.32 & 20.09 & 9.74 \\
\hline
\end{tabular}

Means followed by same letter do not differ by Scott-Knott test $(P \leq 0.05)$. 
For drought tolerance, among the 42 hybrids TSxPT evaluated in the years 2007 and 2010, eight were highly susceptible, with average scores ranging from 1.0 to $1.3,11$ had moderately susceptibility (1.5 to 2.1 ), and 23 hybrids were tolerante to drought, with scores varying from 2.2 to 3.0 (Table 2). The Figure 3 illustrated two hybrids, one highly susceptible (TSxPT 26) and the other, (TSxPT 299), that is very tolerant to drought. As in 2010 there was a very marked dry season in the region of the experiment, the plants showed low production (Table 2). The correlation between the scores for drought tolerance and the production of plants of Pera sweet orange grafted on hybrids TSxPT was $0.61(P<0.05)$ (Figure 4). The more susceptible to drought was the rootstock, less productive was the canopy, Pera sweet orange, due to fruit dropping. In the present study, we found a moderate correlation between plant vigor and tolerance to drought. The correlations between the scores for drought tolerance and plant height or canopy volume were 0.43 and 0.45 , respectively. However, the smaller canopy showed the lowest tolerance to drought (Tables 1 and 2). According to Pires et al. (2005), the duration and intensity of drought, initially reduces the size and number of cells and organs, leading to the closure of stomata, reduction of photosynthesis, wilting and drop of leaves and fruit and consequent decrease in production. The architecture of the root system and the ability to explore soil moisture in deep soil layers, along with the relation between scion/rootstock may be an important mechanism of escape to water deficits (Medina et al., 2005). Ford (1954) reports that drought tolerance of citrus grafted on Rough lemon was associated with deeper roots.

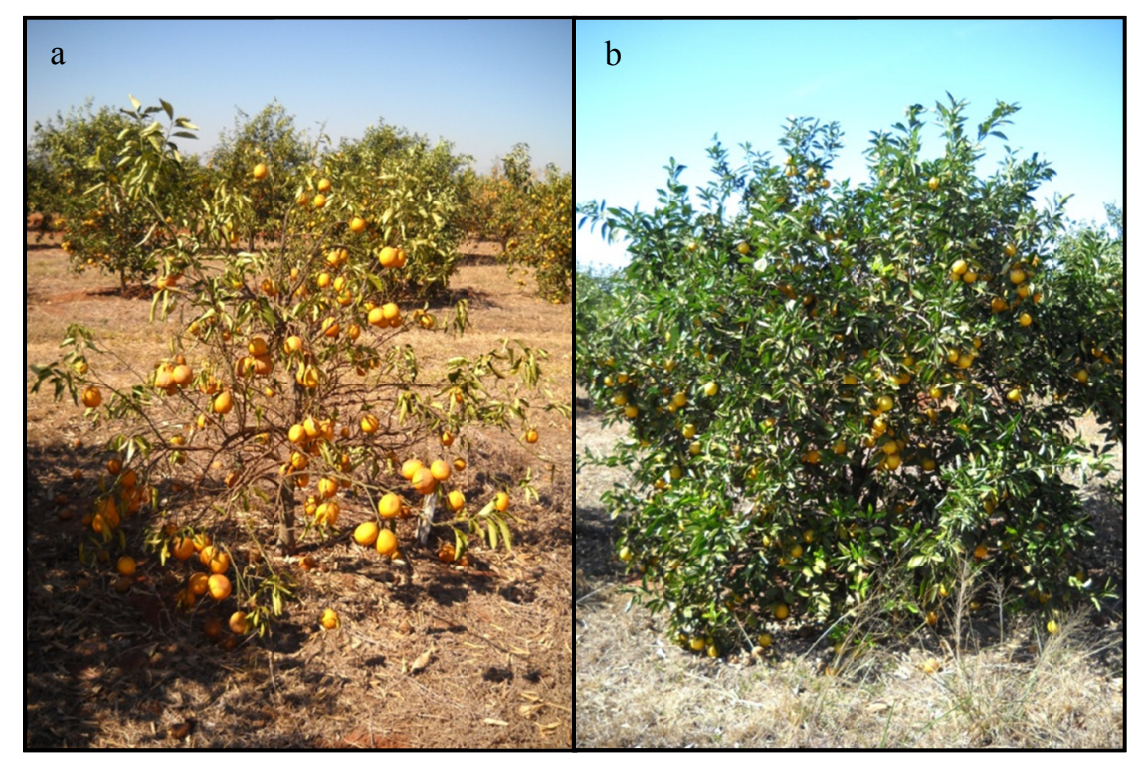

Figure 3. Pera sweet orange grafted on hybrids of Sunki mandarin $\mathrm{x}$ Poncirus trifoliata showing different levels of susceptibility to drought, after 90 days of a dry season. a) TSxPT 26 - high susceptibility (score 1), b) TSxPT 299 resistant (score 3). (Colômbia, SP, Brazil, 2010)

It is known that Pera sweet orange presents incompatibility with various rootstocks as Poncirus trifoliata and its hybrids, Volkamerian lemon (C. volkameriana V. Tennore \& Pasquale) and several selections of Rough lemon ( $C$. jambhiri Lushington) (Pompeu Junior, 2005). Therefore, this experiment evaluated the compatibility of Pera sweet orange with 42 hybrids of Sunki mandarin x Poncirus trifoliata and it was found that two of them (TSxPT 245 and 254) showed the typical symptom of incompatibility (Figure 5), ie, we observed the gum line or necrosis in the region of grafting, similar to that reported by Nauriyal et al. (1958). Cristofani-Yaly et al. (2007) had already observed incompatibility of hybrid TSxPT 245 and 254 with Pera when the plants were four years old.

The characteristic symptom of citrus sudden death, the development of a yellow stain in the phloem part of the rootstock in the region of grafting, observed after removal of part of the cortex (Gimenes-Bassanezi \& Fernandes, 2001), was not observed in any of the hybrids TSxPT evaluated in 2010, when the plants were seven years old. In 2007 , when the plants were four years old, symptoms of citrus sudden death were also not observed in evaluations by Cristofani-Yaly et al. (2007). 


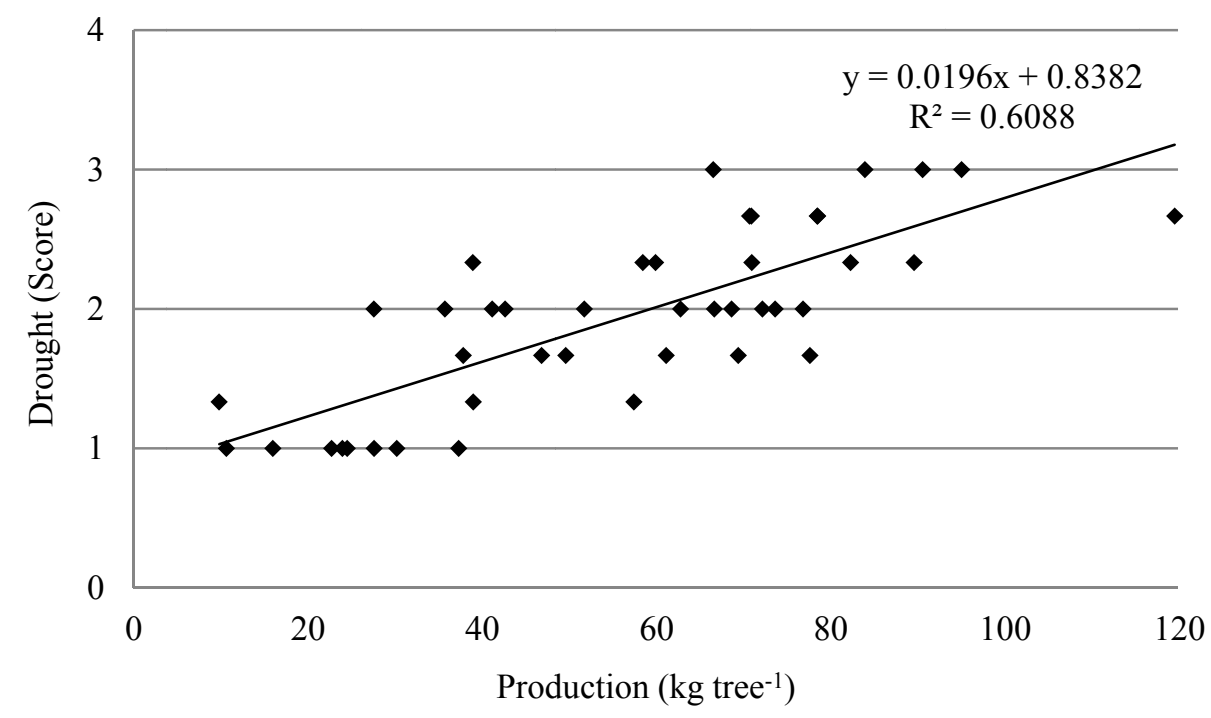

Figure 4. Correlation between the scores of drought and production $\left(\mathrm{kg} \mathrm{tree}^{-1}\right)$ of plants Pera sweet orange grafted on 42 hybrids of Sunki mandarin x Poncirus trifoliata (Colômbia, SP, Brazil, 2010)

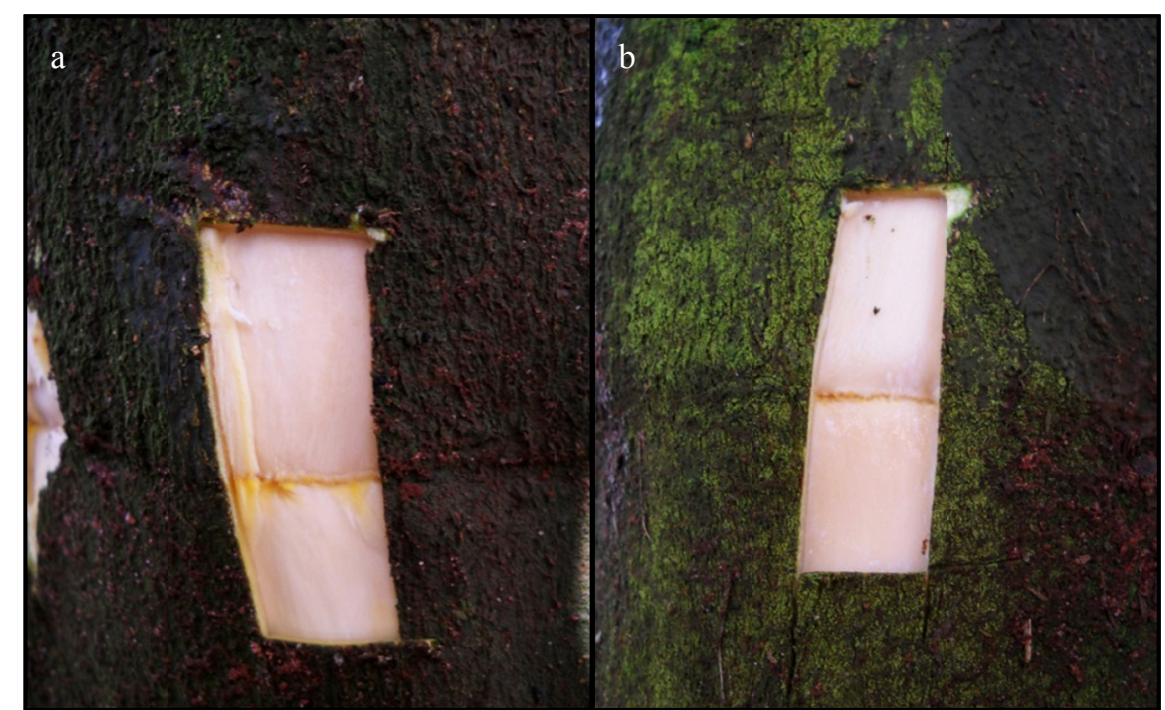

Figure 5. Symptom of incompatibility in the region of grafting between Pera sweet orange with hybrids of Sunki mandarin x Poncirus trifoliata 245 (a) and 254 (b) (Colômbia, SP, Brazil, 2010)

\section{Conclusions}

There were differences in height, diameter and volume of Pera sweet orange grafted on 42 hybrids of Sunki mandarin x Poncirus trifoliata and the hybrids TSxPT 14, 26, 110 and 137 were considered to induce dwarfing to the canopy.

The hybrids TSxPT 155, 142, 54, 92 induced higher production of Pera sweet orange.

Differences were observed in physico-chemical characteristics of the fruit of Pera sweet orange grafted on different TSxPT hybrids.

Different degrees of drought tolerance were observed among the hybrids and TSxPT 245 and 254 showed to be incompatible with Pera sweet orange.

\section{References}

Alencar, J. Podridão do pé dos citros. (1941). Boletim n.6, Escola Superior de Agricultura de Minas Gerais, Viçosa. 
CEPAGRI - Centro de Pesquisas Meteorológicas e Climáticas Aplicadas à Agricultura. (2011). Retrieved January 04, 2011, from http://www.cpa.unicamp.br

Bitancourt, A. A. (1940). A doença dos citros no Vale do Paraíba. O Biológico, 6, 268-269.

Blumer, S., \& Pompeu Junior, J. (2005). Avaliação de citrandarins e outros híbridos de trifoliata como porta-enxertos para citros em São Paulo. Revista Brasileira de Fruticultura, 27(2), $264-267$. http://dx.doi.org/10.1590/S0100-29452005000200019

Cristofani-Yaly, M., Bastianel, M., Faldoni, L., Blumer, S., Pompeu Junior, J., Campos, T. M. P., \& Machado, M. A. (2007). Seleção de citrandarins (tangerina Sunki vs Poncirus trifoliata) para porta-enxertos de citros. Laranja, 28(1-2), 71-79.

Di Giorgi, F., Ide, B. Y., Dib, K., Marchin, R. J., Triboni, H. R., \& Louro, R. W. (1990). Contribuição ao estudo do comportamento de algumas variedades de citros e suas implicações agro-industriais. Laranja, 11(2), $567-612$.

Di Giorgi, F., Ide, B. Y., Dib, K., Marchin, R. J., Triboni, H. R., \& Wagner, R. L. (1994). Proposta para ampliação do conceito de estimativa de safra quantitativa para qualitativa. Laranja, 15(2), 97-122.

Ferreira, D. F. (2008). SISVAR: um programa para análises e ensino de estatística. Revista Symposium, 6, 36-41.

Ford, H. W. (1954). The influence of rootstock and tree age on root distribution of citrus. Proc. Am. Soc. Hortic. Sci., 63, 137-142.

Gimenes-Fernandes, N., \& Bassanezi, R. B. (2001). Doença de causa desconhecida afeta pomares cítricos no Norte de São Paulo e no Sul do Triângulo Mineiro. Summa Phytopathologica, 27(1), 93.

Levitt, J. (1980). Responses of plant to environmental stresses. II. Water, radiation, salt, and other stresses. New York: Academic Press.

Medina, C. L., Rena, A. B., Siqueira, D. L., \& Machado, E. C. (2005). Fisiologia dos citros. In D. Mattos Junior, J. D. De Negri, R. M. Pio, \& J. Pompeu Junior (Eds.), Citros (1st edn). Cordeirópolis, SP: Centro APTA Citros Sylvio Moreira, 1, 147-195.

Mendel, K. (1956). Roostock-scion relationships in Shamouti trees on light soil. Katavim: records of the agricultural research station, 6, 35-38.

Nauriyal, J. P., Shannon, L. M., \& Frolich, E. F. (1958). Eureka lemon trifoliate orange: incompatibility. Journal of the American Society for Horticultural Science, 72, 273-283.

Ortolani, A. A., Júnior, M. J. P., \& Alfonsi, R. R. (1991). Agroclimatologia e cultivos dos citros. In O. Rodriguez, F. Viegas, J. Pompeu Júnior, \& A. A. Amaro (Eds.), Citricultura Brasileira. Campinas: Fundação Cargill, 1, 153-194.

Pires, R. C. M., Luchiari, D. J. F., Arruda, F. B., Mossak, I. (2005). Irrigação. In D. Mattos Junior, J. D. De Negri, R. M. Pio, J. Pompeu Junior (Eds). Citros. 1. ed. Cordeirópolis, SP: Centro APTA Citros Sylvio Moreira, 1, 369-408.

Pompeu Junior, J. (2001). Porta-enxertos para citros potencialmente ananicantes. Laranja, 22(1), 147-155.

Pompeu Junior, J. (2005). Porta-enxertos. In: Mattos Junior, D.; De Negri, J.D.; Pio, R.M.; Pompeu Junior, J. (Eds). Citros. 1. ed. Cordeirópolis, SP: Centro APTA Citros Sylvio Moreira, 1, 63-104.

Pompeu Junior, J., \& Blumer, S. (2011). Citrandarins e outros híbridos de trifoliata como porta-enxertos para laranjeira Valência. Citrus Research \& Technology, 32(3), 133-138.

Pompeu Junior, J., \& Blumer, S. (2009). Híbridos de trifoliata como porta-enxertos para a laranjeira Valência. Pesquisa Agropecuária Brasileira, 44(7), 701-705. http://dx.doi.org/10.1590/S0100-204X2009000700008

Rodriguez, O., Rossetti, V. V., Muller, G. W., Moreira, C. S., Prates, H. S., Negri, J. D., \& Greve, A. (1979). Declínio de plantas cítricas em São Paulo. In CONGRESSO BRASILEIRO DE FRUTICULTURA, 5., 1979, Pelotas. Anais... Pelotas: Sociedade Brasileira de Fruticultura, 927-932.

Scott, A. J., \& Knott, M. (1974). Acluster analysis method for grouping means in the analysis of variance. Biometrics, 30(2), 507-512.

Viégas, F. C. P. (1991). A industrialização dos produtos cítricos. In O. Rodriguez, F. Viegas, J. Pompeu Júnior, \& A. A. Amaro (Eds.), Citricultura brasileira. Campinas: Fundação Cargill, 2, 898-921. 